\title{
ADESÃO A UM GRUPO EDUCATIVO DE PESSOAS PORTADORAS DE DIABETES MELLITUS: PORCENTAGEM E CAUSAS
}

\author{
ADHERENCE OF PEOPLE WITH DIABETES MELLITUS TO AN \\ EDUCATIONAL GROUP: PERCENTAGE AND CAUSES
}

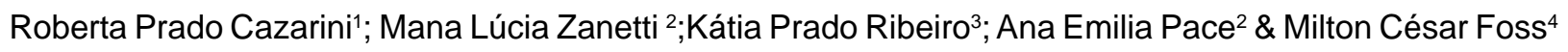

\begin{abstract}
${ }^{1}$ Escola de Enfermagem de Ribeirão Preto-USP. ${ }^{2}$ Docente. EERP-USP. ${ }^{3}$ Enfermeira, bolsista de Apoio Técnico à Pesquisa do CNPq. ${ }^{4}$ Docente. Faculdade de Medicina de Ribeirão Preto-USP.

Correspondëncia: Profa Dra Maria Lúcia Zanetti - Departamento de Enfermagem Geral e Especializada da EERP-USP; Avenida Bandeirantes, 3900 - Campus Universitàrio - CEP 14040-902 — Ribeirão Preto/SP — FAX: 633-3271/630-2561 zanetti@eerp.usp.br.
\end{abstract}

CAZARINI RP; ZANETTI ML; RIBEIRO KP; PACE AE \& FOSS MC. Adesão a um grupo educativo de pessoas portadoras de diabetes mellitus: porcentagem e causas. Medicina, Ribeirão Preto, 35: 142-150, abr./ jun. 2002

RESUMO: Este estudo descritivo teve como objetivo identificar o perfil de pessoas diabéticas e analisar suas dificuldades em participar de um grupo de educação para diabéticos. Foram entrevistados 66 diabéticos, atendidos num hospital de grande porte, no período de maio a junho de 2000. Os resultados apontam que, dos 66 (100\%) sujeitos investigados, 60,6\% sabiam da existência de atividade educativa no hospital, mas apenas $21,2 \%$ participam das atividades educativas oferecidas. Dos que sabiam da existência de atividade educativa, as principais causas mencionadas para a não participação foram: a falta de interesse, horário inadequado e dificuldades de transporte. Com isso, fica demonstrada a necessidade de fortalecimento e ampliação do grupo de educação para diabéticos.

UNITERMOS: Diabetes Mellitus educação; enfermagem.

\section{INTRODUÇÃO}

A importância de planejar grupos de educação para pessoas portadoras de diabetes justifica-se, pois, apesar dos grandes avanços tecnológicos em relação ao diagnóstico e ao tratamento, um alto percentual delas não adere ao tratamento preconizado.

Com a educação dos portadores de diabetes, é possível conseguir reduções importantes das complicações e conseqüente melhoria da qualidade de vida , porque entendemos que a educação para a saúde, feita por grupos especializados, poderá ajudar os profissionais, pessoas portadoras de diabetes e famílias a atingirem a qualidade de vida, ao longo do processo de doença. Como a educação para a saúde é uma tarefa que requer conhecimentos, dedicação e persistência, é de responsabilidade de cada integrante da equipe de saúde. Como parte essencial do tratamento, constituise num direito e num dever do paciente e dos profissionais responsáveis pela promoção da saúde.

O processo de educação deve acontecer de forma gradativa, contínua, interativa e adequada, considerando-se as características do educando ${ }^{2}$ e através de atendimento individual ou realização de grupos com outros pacientes, na sala de espera; de programação de visitas domiciliares; em colônia de férias, cursos regulares ou associações de diabéticos, o que favorecerá a compreensão do diabetes pela pessoa acometida por ele, visando ao seu tratamento e controle.

A nossa experiência com grupos de educação para pessoas portadoras de diabetes, em um hospital 
grande porte do Interior Paulista, tem demonstrado, particularmente, que os participantes beneficiam-se da interação com outras pessoas que enfrentam os mesmos problemas, encontrando não só apoio emocional, mas, também, idéias e sugestões para seu novo estilo de vida. Assim, trabalhar em grupo tem possibilitado o desenvolvimento de destrezas, aliviado as pressões e temores da pessoa, economizado tempo e esforço do educador.

Reconhecendo os benefícios que a educação em diabetes poderá trazer às pessoas, através do planejamento das atividades em grupo, ajudando-as no tratamento e controle da doença, não compreendemos por que é baixa a adesão às atividades educativas, realizadas no Ambulatório de Endocrinologia e Metabologia do Hospital das Clínicas da Faculdade de Medicina de Ribeirão Preto da Universidade de São Paulo (HCFMRP-USP), que atende uma média de 350 pacientes/mês e conta a participação de apenas 20 pacientes no grupo de educação. Diante dessa questão, elaboramos este estudo, que tem como objetivos: de terminar o perfil das pessoas adultas portadoras de diabetes dos tipos 1 e 2 , atendidas num hospital de grande porte e analisar as dificuldades das pessoas diabéticas em participar das atividades do grupo de educação para diabéticos.

\section{MATERIAL E MÉTODOS}

\subsection{Delineamento do estudo}

Utilizou-se o método descritivo.

\subsection{Local de estudo}

Realizou-se no Ambulatório de Endocrinologia e Metabologia do HCFMRP-USP.

\subsection{População e amostra}

No ambulatório de Endocrinologia e Metabologia do referido hospital, estão em seguimento regular aproximadamente 700 pacientes com diabetes, retornando, durante o ano, de três a cinco vezes para consultas médicas, de acordo com as necessidades de cada um. A amostragem foi por conveniência, tendo como critério o período de 15 de maio a 17 de julho de 2000. Assim, a amostra foi constituída por 66 pessoas adultas, com diabetes dos tipos 1 e 2 .

\subsection{Variáveis do estudo}

Variáveis sociodemográficas (sexo, faixa etária, escolaridade, procedência, ocupação e renda familiar mensal), variáveis relacionadas ao diagnós- tico e tratamento, (tipo de diabetes, tempo de doença, tipo de tratamento, índice de massa corporal (IMC) e exames laboratoriais) e as variáveis relacionadas à participação ou não dos pacientes no grupo, onde consideramos as dificuldades e as facilidades referidas por eles, decorrentes de sua participação nas atividades educativas.

\subsection{Elaboração do instrumento de coleta de dados}

Constituiu-se de roteiro sistematizado, considerando-se as variáveis do estudo, a experiência pessoal do pesquisador e a revisão da literatura. Esse instrumento com três partes, contém dezesseis questões fechadas e quatro abertas.

\subsection{Validação de instrumento}

Solicitou-se a colaboração de três especialistas em diabetes, para apreciarem o instrumento quanto à clareza dos itens, facilidade de leitura, compreensão e forma de apresentação, tendo o estudo-piloto sido realizado no período de 24 de abril a 08 de maio de 2000, com cinco pessoas diabéticas.

\subsection{Coleta de dados}

Obtiveram-se os dados das variáveis sociodemográficas e das relacionadas à participação ou não dos pacientes do grupo mediante entrevistas dirigidas, realizadas às segundas-feiras, antes ou após a consulta médica do paciente, ocasião em que o pesquisador registrou, no instrumento, os dados colhidos. Os dados referentes às variáveis relacionadas ao diagnóstico e ao tratamento foram obtidos mediante consulta ao prontuário do paciente.

\subsection{Organização dos dados para análise}

Os dados contidos no formulário receberam códigos específicos, quando selecionamos cada variável que era transportada para uma planilha. Montamos a estrutura do banco de dados a partir das planilhas, sendo o mesmo formatado no programa Word (para Windows 95, versão 6.0). A análise dos dados obedeceu às normas preconizadas para um estudo descritivo, uma vez que tomamos por base a literatura referente à temática estudada.

\subsection{Aspectos éticos}

Submeteu-se o estudo à análise do Comitê de Ética em Pesquisa do HCFMRP-USP, esclarecemos aos sujeitos participantes os objetivos e a natureza da investigação e iniciamos as entrevistas, após eles terem assinado o termo de consentimento. 


\section{RESULTADOS}

Dos $66(100 \%)$ da amostra estudada, $17(25,8 \%)$ são do sexo masculino e $49(74,2 \%)$ do feminino. (Ta bela I).

Em relação à faixa etária, observamos uma variação de 22 a 88 anos, com uma média de 50 anos de idade. Podemos verificar que a maioria $68,2 \%$ portadores de diabetes tem de 41 a 90 anos. Quanto à sua procedência, 29 (43,9\%) são de Ribeirão Preto, 31 (47\%) provenientes de outros municípios do Estado de São Paulo e $6(9,1 \%)$ procedem de outros esta- dos do país. (Tabela I).

Ao analisarmos a escolaridade, verificamos que a maioria $(95,5 \%)$ dos portadores de diabetes mellitus é alfabetizada.

No que se refere à ocupação dos portadores de diabetes, vimos que 22 (33,3\%) são aposentados. $20(30,3 \%)$ são mulheres e se dedicam ao lar e 10 $(15,2 \%)$, trabalhadores assalariados, administrativos, técnicos e científicos. Quanto à renda familiar dos portadores de diabetes, observamos que $41(62,1 \%)$ deles possuem renda familiar entre um e três salários mínimos. (Tabela I).

\begin{tabular}{|c|c|c|}
\hline Sexo & $\mathrm{N}^{\mathrm{w}}$ & $\%$ \\
\hline $\begin{array}{l}\text { Masculino } \\
\text { Ferriximo }\end{array}$ & $\begin{array}{l}17 \\
49\end{array}$ & $\begin{array}{l}25,8 \\
74,2\end{array}$ \\
\hline Faixa etária & $\mathbf{N}^{\mathbf{r}}$ & $\%$ \\
\hline $\begin{array}{l}22 /-30 \\
31 /-40 \\
41 /-50 \\
51 /-60 \\
61 /-70 \\
71 /-90\end{array}$ & $\begin{array}{r}12 \\
9 \\
12 \\
18 \\
11 \\
4\end{array}$ & $\begin{array}{r}18,2 \\
13,6 \\
18,2 \\
27,2 \\
16,6 \\
6,2\end{array}$ \\
\hline Procedê ncia & $\mathrm{N}^{\mathrm{N}}$ & $\%$ \\
\hline $\begin{array}{l}\text { Ribeirão Preto } \\
\text { Ouros Muricipios } \\
\text { Outros Estados }\end{array}$ & $\begin{array}{r}29 \\
31 \\
6\end{array}$ & $\begin{array}{r}43,9 \\
47,0 \\
9,1\end{array}$ \\
\hline $\begin{array}{l}\text { Sem escolaridade/alfabetizado } \\
\text { Sem escolaridade/ analfabeto }\end{array}$ & $\begin{array}{l}3 \\
3\end{array}$ & $\begin{array}{l}4,5 \\
4,5\end{array}$ \\
\hline Octpação & $\mathrm{N}^{\mathrm{r}}$ & $\%$ \\
\hline $\begin{array}{l}\text { Profissionais liberais autônomos } \\
\text { Trabathadores assalariados admiristrativos técricos e ciertificos } \\
\text { Empregados domésticos ubanos } \\
\text { Aposertados } \\
\text { Estudartes } \\
\text { Do lar } \\
\text { Desertquegado }\end{array}$ & $\begin{array}{r}5 \\
10 \\
1 \\
22 \\
1 \\
20 \\
7\end{array}$ & $\begin{array}{r}7,6 \\
15,2 \\
1,5 \\
33,3 \\
1,5 \\
30,3 \\
10,6\end{array}$ \\
\hline Renda Familiar & $\mathrm{N}^{\mathrm{w}}$ & $\%$ \\
\hline $\begin{array}{l}\text { Sem renda } \\
1,0 / 3,5 \\
3,5 / 6,5 \\
6,5 / 10,0 \\
10,0 /-14,0 \\
+ \text { de } 14,0\end{array}$ & $\begin{array}{r}2 \\
41 \\
8 \\
4 \\
6 \\
5\end{array}$ & $\begin{array}{r}3,0 \\
62,1 \\
12,2 \\
6,0 \\
9,1 \\
7,6\end{array}$ \\
\hline
\end{tabular}


Em relação à composição familiar dos portadores de diabetes, verificamos que dos 66 participantes deste estudo, 21 (31.8\%) moram com mais uma pessoa, $17(25,8 \%)$ com mais três e $12(18,2 \%)$ com mais duas.

Em relação ao tipo de diabetes, verificamos que a maioria dos pacientes estudados, 38 (57,6\%), tem diabetes do tipo 1 e $28(42,4 \%)$, do tipo 2 .

Ao analisar o tempo em que os portadores de diabetes (Tabela II) convivem com a doença, verificamos que $22(33,5 \%)$ têm a doença entre dois e cinco anos. A maioria (59\%) apresenta a doença há mais de cinco anos.

Na Tabela II, observamos que $10,6 \%$ dos pacientes fazem o tratamento somente com medicamentos e $33,3 \%$ com medicamento e dieta, porém para que o tratamento do diabetes mellitus dos tipos 1 e 2 obtenha um bom controle metabólico, é necessária a tríade: dieta, medicamentos e exercícios físicos. Podemos observar, ainda, que $44(66,7 \%)$ possuem Índi- ce de Massa Corporal (IMC) maior que 25,0, apresentando sobrepeso. Em relação à hipertensão arterial, verificamos que $37(56,1 \%)$ apresentam a doença.

Ao analisar os resultados da glicose plasmática de jejum, constatamos que $47(71,2 \%)$ pacientes apresentavam glicemia superior a $110 \mathrm{mg} / \mathrm{dl}$. Constatamos que os exames de $14(21,2 \%)$ sujeitos mostraram variação da hemoglobina glicosilada menor ou igual a 8,$0 ; 32(48,5 \%)$, entre 8,1 a 10 e $20(30,3 \%)$, maior que 10 .

Quanto ao HDL, obtivemos que $26(39,4 \%)$ sujeitos apresentaram maior que $45 \mathrm{mg} / \mathrm{dl} ; 01(1,5 \%)$, igual a $45 \mathrm{mg} / \mathrm{dl}$ e $39(59,1 \%)$ menor que $45 \mathrm{mg} / \mathrm{dl}$. Em relação aos níveis de colesterol total, constatamos que $41(62,1 \%)$ deles estavam com o valor dentro dos limites normais, $14(21,2 \%)$, entre 200 a $239 \mathrm{mg} / \mathrm{dl} \mathrm{e}$ $11(16,7 \%)$, maior que $240 \mathrm{mg} / \mathrm{dl}$. Quanto aos triglicérides, $34(51,5 \%)$ apresentam resultados menores que $150 \mathrm{mg} / \mathrm{dl}, 14$ (21,2\%), entre 150 e $199 \mathrm{mg} /$ dl e $18(27,3 \%)$ maiores que $200 \mathrm{mg} / \mathrm{dl}$. (Tabela III)

\begin{tabular}{|c|c|c|}
\hline Te mpo da doença (em anos) & $\mathrm{N}^{0}$ & $\%$ \\
\hline $\begin{array}{l}<2 \text { anos } \\
2 \text { a } 5 \text { anos } \\
6 \text { a } 10 \text { anos } \\
11 \text { a } 16 \text { anos } \\
>16 \text { anos }\end{array}$ & \begin{tabular}{r|}
5 \\
22 \\
16 \\
8 \\
15
\end{tabular} & $\begin{array}{r}7,5 \\
33,5 \\
24,0 \\
12,0 \\
23,0\end{array}$ \\
\hline Típo de tratamento & $\mathrm{N}^{\mathrm{D}}$ & $\%$ \\
\hline $\begin{array}{l}\text { Somente dieta } \\
\text { Somente antidiabético oral } \\
\text { Somente instlina } \\
\text { Dieta e antidiabético oral } \\
\text { Dieta e irsulina } \\
\text { Dieta, antidiabético oral e exercícios } \\
\text { Dieta, insulina e exercícios } \\
\text { Artidiabético oral e exercícos } \\
\text { Artidiabético oral, instlina, dieta e exercícios }\end{array}$ & $\begin{array}{r}1 \\
3 \\
4 \\
8 \\
14 \\
10 \\
23 \\
2 \\
1\end{array}$ & $\begin{array}{r}1,5 \\
4,5 \\
6,1 \\
12,1 \\
21,2 \\
15,2 \\
34,8 \\
3 \\
1,5\end{array}$ \\
\hline Índice de massa corporal (IM C) & $\mathrm{N}^{\mathrm{0}}$ & $\%$ \\
\hline $\begin{array}{l}<18,5 \\
18,5 \text { a } 24,9 \\
25,0 \text { a } 29,9 \\
30,0 \text { a } 34,9 \\
35,0 \text { a } 39,9 \\
>40\end{array}$ & $\begin{array}{l}02 \\
20 \\
23 \\
11 \\
05 \\
05\end{array}$ & $\begin{array}{r}3,0 \\
30,3 \\
34.8 \\
16,7 \\
7,6 \\
7,6\end{array}$ \\
\hline
\end{tabular}




\begin{tabular}{|c|c|c|}
\hline \multicolumn{3}{|c|}{ 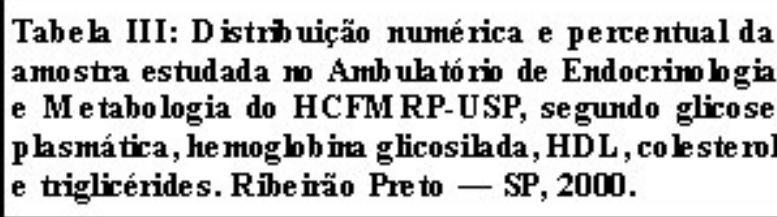 } \\
\hline Glicose plasmática (em mg/dI) & $\mathrm{N}^{0}$ & $\%$ \\
\hline $\begin{array}{c}<110 \\
110 \text { a } 140 \\
>140\end{array}$ & $\begin{array}{r}19 \\
11 \\
36\end{array}$ & $\begin{array}{l}28,8 \\
16,7 \\
54,5\end{array}$ \\
\hline Hemogbbina glicosiada & $\mathrm{N}^{\mathrm{a}}$ & $\%$ \\
\hline $\begin{array}{c}\text { Menot ou igual a } 8 \\
8,1 \text { a } 10 \\
>10\end{array}$ & $\begin{array}{l}14 \\
32 \\
20\end{array}$ & $\begin{array}{l}21,2 \\
48,5 \\
30,3\end{array}$ \\
\hline HDL & $\mathrm{N}^{\mathrm{a}}$ & $\%$ \\
\hline $\begin{array}{l}>45 \\
=45 \\
<45\end{array}$ & $\begin{array}{l}26 \\
01 \\
39\end{array}$ & $\begin{array}{r}39,4 \\
1,5 \\
59,1\end{array}$ \\
\hline Colesterol Total & $\mathrm{N}^{0}$ & $\%$ \\
\hline $\begin{array}{c}<200 \\
200 \text { a } 239 \\
>240\end{array}$ & $\begin{array}{r}41 \\
14 \\
11\end{array}$ & $\begin{array}{l}62,1 \\
21,2 \\
16,7\end{array}$ \\
\hline Triglicé rides & $\mathrm{N}^{0}$ & $\%$ \\
\hline $\begin{array}{c}<150 \\
150 \text { a } 199 \\
>200\end{array}$ & $\begin{array}{l}34 \\
14 \\
18\end{array}$ & $\begin{array}{l}51,5 \\
21,2 \\
27,3\end{array}$ \\
\hline
\end{tabular}

Ao indagarmos aos portadores de diabetes a respeito das atividades educativas realizadas no hospital, verificamos que apenas $40(60,6 \%)$ deles sabiam da existência de alguma atividade educativa. Dentre os que sabiam da existência de alguma atividade educativa tio hospital, 18 (27,3\%) responderam que a atividade desenvolvida era de orientação, 16 (24.2\%) achavam que se tratava de palestra, 5 (7,6\%), de grupo de educação, $3(4,5 \%)$, de outras atividades e $24(36,4 \%)$ não responderam à questão, pois não tinham conhecimento da realização de atividade educativa no hospital.

Entre os portadores de diabetes que informaram conhecer o oferecimento dessa atividade educativa no hospital. $28(42,4 \%)$ responderam que não participam dela, $14(21,2 \%)$ disseram que participam e 24 $(36,4 \%)$ não responderam à questão.

Quando indagamos aos portadores de diabetes por que motivo não participavam da atividade, 27 $(40,9 \%)$ informaram que não sabiam de nenhuma atividade educativa, oferecida pelo hospital. 2 (3\%) não tinham interesse nesse tipo de atividade, $6(9,1 \%)$ achavam o horário inadequado, $4(6,1 \%)$ dependiam de transporte, $7(10,6 \%)$ justificaram que eram de outros municípios, $4(6,1 \%)$ alegaram outros motivos e 16 $(24,2 \%)$ não responderam à questão.

Quando indagamos aos portadores de diabetes sobre sua participação em atividade educativa no hospital, no período da manhã e no dia da entrevista, $4(6,1 \%)$ responderam que participaram, $29(43,9 \%)$ não participaram e $33(50 \%)$ não responderam à questão. Em seguida, questionamos sobre os motivos que os levaram a participar da atividade. Dos 66 participantes, $15(22,7 \%)$ responderam que, com a atividade, aprendiam mais sobre a doença e $51(77,3 \%)$ não responderam à questão.

Quando perguntamos aos portadores de diabetes que não participavam da atividade educativa no hospital se gostariam de participar dela, constatamos que, dos $66,37(56,1 \%)$ responderam que gostariam, $14(21,2 \%)$ que não e $15(22,7 \%)$ não responderam à questão.

\section{DISCUSSÃO}

Ao analisarmos as variáveis sociodemográficas, verificamos que a maioria $(95,5 \%)$ dos sujeitos é alfabetizada. Esse dado pode facilitar o planejamento de grupos educativos, pois a escolaridade é um fator importante em virtude da complexidade das instruções e/ou informações que esses pacientes necessitam obter. A baixa escolaridade pode dificultar a aprendizagem, pois, à medida que aumenta a complexidade da terapêutica, o paciente necessita de habilidades cognitivas mais complexas para manter o seu controle metabólico ${ }^{3}$.

Acreditamos que a educação seja fundamental em todas as fases do tratamento e, independentemente do grau de instrução, o educador deve empregar sempre uma linguagem simples que favoreça o aprendizado, utilizando as experiências dos pacientes, a de facilitar o processo educativo ${ }^{4,5}$.

Considerando que a maioria dos portadores de diabetes mellitus $(63,6 \%)$ é aposentada e do lar, supomos que o tipo de ocupação não os impede de participar dos grupos de educação. Tal dado, por si só, justifica a necessidade de estimular esses pacientes a participarem de grupos de educação em diabetes.

Os dados apontam que a maioria dos portadores de diabetes mellitus recebe de 1 a 3,5 saláriosmínimos, o que, a nosso ver, pode comprometer a adesão do paciente ao tratamento, pois, para a obtenção 
de um bom controle metabólico, são necessários: dieta balanceada, controle domiciliar através de utilização de materiais e aparelhos, bem como o uso adequado de calçados, entre outros.

Em relação à composição familiar, obtivemos que a maioria $(75,8 \%)$ dos sujeitos mora com mais de uma pessoa, o que pode colaborar para a adesão ao tratamento, uma vez que o apoio familiar representa um fator importante no cuidado da pessoa portadora de doença crônica, pois os familiares deverão estar envolvidos no cuidado, visando obter um melhor controle metabólico Concordamos que a família influencia e ajuda no controle do diabetes mellitus, pois, quando acompanhado e apoiado, o paciente apresenta maior adesão ao tratamento e melhora do controle metabólico'.

São enormes os avanços tecnológicos na área de diabetes mellitus, $\mathrm{n}$ entanto, analisando os dados sociodemográficos, observamos que muitos pacientes podem apresentar dificuldades de acesso à tecnologia disponível para um controle estritamente domiciliar. Por outro lado, as atividades em grupos de educação parecem indicar um outro caminho para ajudá-los, pois, mediante tecnologia simplificada, eles podem orientar-se acerca do controle da doença.

Em relação ao tipo de diabetes, acreditamos que o diabetes do tipo 1 tenha apresentado unia porcentagem maior $(57,6 \%)$ talvez porque os portadores desse tipo devam receber acompanhamento contínuo, no hospital em estudo. Ao contrário, os que têm um bom controle metabólico retornam de três a cinco vezes por ano, ao hospital, para consultas médicas, de acordo com as necessidades de cada uni no entanto, como a maioria apresenta um controle metabólico aceitável ou ruim, esses retornos são mais freqüentes. Outro fator que pode ter contribuído para que o portador de diabetes do tipo 1 se mostrasse mais freqüente em nosso estudo, é que o hospital-escola de nível terciário, atende a pacientes de alta complexidade, com complicações crônicas e controle metabólico instável.

Verificamos que 59\% dos pacientes apresentam a doença há mais de cinco anos. A literatura aponta que há relação entre a duração do diabetes mellitus e o surgimento de complicações microvasculares ${ }^{10}$. Assim, o controle intensivo do diabetes mellitus, conduzido por uma equipe multiprofissional, com instrumentalização para realizar um bom seguimento e apoio continuado aos pacientes, tem conseguido efetivamente reduzir a progressão das retinopatias, nefropatias e neuropatias ${ }^{1}$.
No que se refere ao tipo de tratamento, constatamos que $43,9 \%$ dos portadores de diabetes realizam o tratamento, utilizando somente medicamentos e dieta. Para conscientização dessa população, deve ser implementado todo um programa de educação com ênfase na importância do plano alimentar também associado aos benefícios da atividade física, visando à redução dos sintomas, prevenção de complicações agudas e crônicas, redução da mortalidade e melhora da qualidade de vida.

Desse modo, cabe aos enfermeiros intensificar as atividades educativas em grupo, enfatizando a atividade física como parte do tratamento e considerando as dificuldades no que diz respeito ao tipo de diabetes, idade, grau de controle metabólico complicações crônicas, entre outras, de modo a assegurarem que a pessoa possa adquirir conhecimentos e habilidades para cuidar de si mesma.

Considerando que $53 \%$ dos pacientes apresentam sobrepeso e obesidade e que $34,8 \%$ fazem o tratamento com dieta, insulina e exercícios físicos, recomendamos que o grupo de educação dê ênfase à dieta e aos exercícios físicos, visando à redução do peso corporal desses indivíduos, pois isso permite a obtenção de melhores parâmetros em relação à hiperglicemia, hiperlipidemia, hipertensão arterial e proteinúria $^{11,12,13}$.

Verificamos que $56,1 \%$ dos sujeitos são hipertensos. A coexistência de hipertensão arterial e diabetes mellitus multiplica de maneira exponencial o risco de morbidade e mortalidade por problemas relacionados com macro e microangiopatia. Recentemente, estudo realizado no Reino Unido, UKPDS ${ }^{14}$ demonstrou que o controle da glicemia e da pressão arterial em pessoas com diabetes mellitus tipo 2 são eficazes para reduzir o risco de macro e microangiopatia. Assim, as atividades de educação em grupo devem contemplar a monitoração da pressão arterial, levando em consideração os critérios da Organização Mundial da Saúde, de 1999, quais sejam: a redução do peso corporal e do consumo de álcool, abandono do tabagismo, restrição de sal a menos de $3 \mathrm{~g} / \mathrm{dia}$, enfocando, principalmente, o papel dos fármacos, tais como: doses, horários de administração e efeitos colaterais, a fim de promover a adesão do paciente ao tratamento.

Constatamos que $71,2 \%$ dos pacientes apresentaram glicose plasmática de jejum superior a $110 \mathrm{mg}$ / dl. Estudo comparativo entre portadores de diabetes mellitus do tipo 2, que não participavam do mesmo, mostrou redução dos níveis de glicemia e melhor conhecimento sobre o diabetes. ${ }^{15}$ 
Verificamos que $39(59,1 \%)$ dos sujeitos apresentam níveis de HDL e $32(48,5 \%)$ triglicérides elevados, respectivamente. "Sabemos que $80 \%$ das pessoas com diabetes mellitus tipo 2 têm algum tipo de dislipidemia. A freqüência da hipercolesterolemia isolada é igual à da população em geral, porém seu impacto como fator de risco cardiovascular chega a ser duas vezes maior na pessoa com diabetes meilitus. A alteração mais freqüiente é a elevação dos triglicérides, com diminuição do colesterol HDL, demonstrando um fator de risco cardiovascular independente" 16 .

Assim, é imprescindível medidas que visem à modificação dos hábitos de vida. Para tanto, recomendamos: controle adequado da glicemia, otimizar o peso do paciente e incrementar o exercício aeróbico regular. A participação de uma especialista em nutrição, junto ao grupo de educação, poderá ajudar na orientação desses pacientes, visando à redução das dislipidemias.

Sabemos que as complicações crônicas estão relacionadas, diretamente, ao grau de controle metabólico, ou seja, pacientes com um bom controle da doença apresentam menor incidência de complicações em relação àqueles com a doença mal controlada. Assim, o objetivo do tratamento visa melhorar os sintomas relacionados à hiperglicemia, controlar complicações agudas, prevenir ou retardar o aparecimento de complicações crônicas e melhorar a qualidade de vida, reduzindo a mortalidade e comortalidade. Para tanto, as metas para o controle metabólico consistem em monitorar as glicemias pré e pós-prandiais, colesterol total, HDL, triglicérides, IMC e hipertensão arterial.

Os resultados deste estudo apontam que as metas de controle metabólico dos pacientes investigados estão entre aceitáveis e ruins, evidenciando dificuldade para adesão ao tratamento, a fim de atingirem um bom controle metabólico.

Uma enfermidade cronicodegenerativa, tal como o diabetes mellitus, requer tratamento durante toda a vida para se obter um bom controle metabólico. No entanto, para a adesão ao tratamento é imprescindível, dentre vários fatores, a atitude de aceitação do paciente frente ao manejo do diabetes.

Um programa de educação nutricional para portadores de diabetes mellitus, apontou, dentre os efeitos satisfatórios redução no uso de hipoglicemiantes e na frequiência de sintomas associados ao diabetes e aumento subjetivo do bem-estar, o que fez com que aumentasse a adesão do paciente ao mesmo ${ }^{17}$.
As dificuldades apresentadas pelos pacientes para seguirem as orientações do grupo de educação de diabetes vão além dos aspectos cognitivos, se levarmos em conta as necessidades individuais e os aspectos biopsicossociais de cada um.

A compreensão e a memorização de conceitos acerca do diabetes mellitus, aplicados em dez grupos de pacientes diabéticos, mostraram que somente um grupo apresentou índice de acerto considerado adequado, comprovando que os pacientes precisavam receber reforços dos diversos conteúdos ensinados durante a atividade educativa ${ }^{11}$.

Para os portadores de diabetes que participavam do grupo, questionamos o que mais gostavam no grupo, do que não gostavam, se tinham alguma sugestão para o grupo e qual foi a contribuição do grupo para com eles.

A maioria dos participantes respondeu que gostava de receber informações sobre a doença, dos exercícios realizados e da troca de experiências.

Sobre as sugestões que teriam para o grupo, um participante respondeu que gostaria de assistir a palestras, com fitas de vídeo, explicando mais sobre o diabetes; um outro sugeriu falar sobre os perigos que o diabetes pode trazer.

Quando indagados sobre a contribuição do grupo, a maioria dos participantes respondeu que aprendeu muito sobre a doença e que os exercícios ajudavam a melhorar a tensão. Outros responderam que o grupo não ajudou muito, pois possuíam bastante conhecimento sobre a doença.

Diante dos dados obtidos neste estudo, podemos perceber que os portadores de diabetes mellitus sabem que a instituição estudada oferece a atividade educativa. No entanto, quando investigamos que tipo de atividade era oferecida, essa população mostrou dificuldade para especificá-la. Isso pode demonstrar a fragilidade do programa de educação, que ainda se encontra sem uma definição clara dos propósitos a serem atingidos. Preocupou-nos, também, saber que $36,4 \%$ do sujeitos desconhecem a oferta dessa atividade educativa, indicando lacunas na divulgação das atividades desenvolvidas no referido hospital.

Como 60 dos pacientes mencionaram conhecer o oferecimento de atividade educativa, causounos inquietação que um número tão alto $(42,4 \%)$ tivesse referido não participar dela. Dentre os motivos apresentados, observamos que eles são, principalmente, de ordem econômica e institucional. Entendemos que a reestruturação do grupo educativo, considerando as 
variáveis apontadas pelos sujeitos e a divulgação das atividades oferecidas, possibilitará urna maior adesão dos portadores de diabetes junto ao grupo de educação, porém vale ressaltar que, atualmente, o referido hospital conta com recursos humanos (enfermeiro e auxiliar de enfermagem) para o desenvolvimento do grupo, com vistas a obter resultados eficazes.

A participação do enfermeiro, na equipe multiprofissional no plano de controle e na prevenção do diabetes, aponta o desconhecimento desses profissionais sobre os recursos que a instituição pode oferecer ao paciente e família ${ }^{18}$.

No atendimento ambulatorial interdisciplinar, a atuação da equipe é um fator diferenciado para a educação e adesão do paciente diabético ao tratamento 19.

Quando indagamos os motivos que os levaram a participar da atividade, chamou nossa atenção que, além de mencionarem o aprendizado sobre o que é o diabetes mellitus, o que é e como deve ser aplicada a insulina, rodízio nos locais de aplicação, entre outros referiram-se também às seguintes atividades grupais: momento de descontração, troca de experiências, estabelecimento de novas amizades, entre outros.

Os grupos, de um modo geral, têm sido bem aceitos pelos pacientes portadores de diabetes mellitus, pois seus participantes beneficiam-se da interação com outras pessoas que enfrentam os mesmos problemas e encontram ali não só apoio emocional, mas, também, idéias e sugestões para modificarem seu estilo de vida. $\mathrm{O}$ grupo ainda permite ao paciente o desenvolvimento de destrezas, alívio das pressões e temores, economizando tempo e esforços do educador ${ }^{20}$.
Assim, acreditamos que um programa educativo com atividades em grupo para pacientes com diabetes mellitus, visando ao autoconhecimento e à autotransformação, passa pelo processo de construção de mundo de cada um deles, propiciando que educador e educando permutem informações, habilidades e atitudes através do vínculo e do compromisso de cada um com o processo em questão.

\section{CONCLUSÕES}

O conjunto de dados obtidos nesta investigação apontam que, dos $66(100 \%)$ portadores de diabetes investigados, $60,6 \%$ deles sabiam da existência de alguma atividade educativa no hospital, e apenas 21 participam das atividades educativas oferecidas. As principais causas mencionadas para a não participação foram a falta de interesse, horário inadequado e dificuldade de transporte.

Os dados indicam que progressos nessa área ainda não se concretizaram, uma vez que os pacientes revelaram que não se sentiam motivados nem reconheciam a educação como um instrumento capaz de ajudá-los a obter um melhor controle metabólico. Portanto, é necessário que haja fortalecimento e ampliação do grupo de educação em diabetes, no referido hospital, com vistas a motivar o paciente e sua família a participarem efetivamente dele, através do qual poderão elaborar seus problemas.

Para tanto, confeccionamos um manual educativo com a finalidade de divulgar as atividades em grupo, desenvolvidas no HCFMRP-USP, para motivar o paciente e sua família a participarem das mesmas.

CAZARINI RP; ZANETTI ML; RIBEIRO KP; PACE AE \& FOSS MC. Adherence of people with Diabetes Mellitus to an educational group: percentage and causes. Medicina, Ribeirão Preto, 35: 142-150, april/june, 2002.

ABSTRACT: This descriptive study aimed at identifying the profile of diabetic people and analyzing their difficulties to participate in an educational group for diabetes. Sixty-six diabetic people assisted in a large hospital from May to June, 2000 were interviewed. Results showed that of the $66(100 \%)$ investigated subjects, $60.6 \%$ were aware of the educational activities in the hospital and that only $21.2 \%$ participated in them. The main reasons for not participating mentioned by those who knew about the activities were: lack of interest, inadequate time and transportation difficulties, which evinces the need to strengthen and enlarge the educational group for diabetes.

UNITERMS: Diabetes_Mellitus;_education;_nursing. 


\section{REFERÊNCIAS BIBLIOGRÁFICAS}

1 - DIABETES CONTROL AND COMPLICATIONS TRIAL RE SEARCH GROUP. The effect of intensive treatment of diabetes on the development and progression of long-term complications in insulin-dependent diabetes mellitus. N Engi J Med 329: 977-986, 1993.

2- SOCIEDADE BRASILEIRA DE DIABETES. Consenso brasileiro de conceitos e condutas para o diabetes mellitus recomendações da Sociedade Brasileira de Diabetes para a prática clínica - 1997. São Paulo, 1997.

3- FOSS MC; PACOLLA GMF; SOUZA NV \& YAZIGI N. Estudo analítico de uma amostra populacional de diabéticos tipo 2 da região de Ribeirão Preto (SP). Rev Assoc Med Bras 35:179-183, 1989.

4 - ROCHA M PS. Assistência de enfermagem em uma unidade de cuidados terciários ao diabético. Rev Bras Enferm 37:182187, 1984.

5 - BURGOS MGPA \& CABRAL PC. Aspectos peculiares da dieta do diabético. In: VILLAR L, coord. Endocrinologia clínica. MEDSI, Rio de Janeiro, p. 363-367, 1999.

6- LUCE M; PADILHA MI; ALMEIDA RLV \& SILVA MO. O preparo para o autocuidado do cliente diabético e família. Rev Bras Enferm 25: 137 - 152, 1991.

7- PADILHA MICS; CARVALHO MTC; SILVA MO \& PINTO VT. Visita domiciliar. Uma alternativa assistencial. Rev Enferm UERJ 2: 83-90, 1994.

8 - COSTAAA \& ALMEIDA NETO JS. Manual de diabetes. Alimentação, medicamentos e exercícios. 3 ed. Sarvier, São Paulo, 1998.

9- VALENCIAGA JLR; GONZALEZ FV; PONS PB \& SANCHEZ $0 \mathrm{~V}$. Repercusion en el control de la diabetes mellitus de una técnica educativa dirigida a la familia. Rev Cubana 11.144 149, 1995.

10- FOSS MC; EIZIRIK DL; PIMENTAWP; SAAD MA; PACOLLA GMF; SOUZA NV \& YAZIGI, N. Estudo analitico de uma amostra populacional de diabéticos insulino-dependentes tipo 1. Arq Bras Endocrinol Metab 30:100-102, 1986.
11 - ALMEIDA HGG; TAKAHASHI OC; HADDAD MCL; GUARIENTE MHDM \& OLIVEIRA ML. Avaliação dos conhecimentos teóricos dos diabéticos de um programa interdisciplinar. Rev Latinoam Enfermagem 2: 145-164, 1995.

12- MILECH A. Perspectivas no tratamento do diabetes meilitus. Diabetes Metab, São Paulo, 2: 170 - 171, 1998.

13- SCHEEN AJ. Programa agressivo para redução de peso no tratamento do diabetes tipo 2. Diabetes Metab, São Paulo, 2:190-201, 1998

14- UK PROSPECTIVE DIABETES STUDY GROUP (UKPDS) Intensive blood-glucose control with sulphonylureas or insulin compared with conventional treatment and risk of complications in patients with type 2 diabetes (UKPDS33) Lancet 352: 837-853, 1998

15- BURCIAGA L; RODRIGUEZ MM \& GUERERO RF. Eficácia de la educación en el control de la glucemia de pacientes con diabetes tipo 2. Rev Med IMSS 37: 39-44, 1999.

16- GUIAS. ALAD DE DIAGNÓSTICO, CONTROLY TRATAMENTO DE LA DIABETES MELLITUS TIPO 2. Dislipidemias. Rev Assoc Latinoam Diabetes, p. 159-161, 2000 Supl. 1.

17- MOTTA DG. A educação participante no controle metabólico e qualidade de vida de mulheres com diabetes meilitus tipo

2. São Paulo, 243 p. Tese de Doutorado, Faculdade de Saúde Pública da USP, São Paulo, p. 1-243, 1998.

18- SILVA MO; DIAS ZP; ALMEIDAAL; FILGUEIRAS ML. Participação do enfermeiro na equipe multiprofissional do plano de educação, controle e prevenção do diabetes meilitus no HUCFF-RJ. Rev Bras Enferm 2/3:133-144, 1992.

19- TAKAHASHI OC; HADDAD MCL; GARIENTE MHDM; ALMEIDA HGG; SCARINCI IC \& TAKAHASHI 1. Atendimento ambulatorial ao paciente diabético. Acta Paul Enferm, São Paulo, 6: 43-47, 1993.

20 - ZANETTI ML; PACE AE \& SOUZA CR. Grupos informativos para pacientes diabéticos. Arq Bras de Endocrinol Metab 43: S182, 1999, Supl.1.

Recebido para publicação em 15/05/2001

Aprovado para publicação em: 28/06/2002 